\title{
Categorical Symmetries at Criticality
}

\author{
Xiao-Chuan $\mathrm{Wu},{ }^{1}$ Wenjie $\mathrm{Ji}^{1}{ }^{1}$ and Cenke $\mathrm{Xu}^{1}$ \\ ${ }^{1}$ Department of Physics, University of California, Santa Barbara, CA 93106
}

(Dated: December 9, 2020)

\begin{abstract}
We study the concept of "categorical symmetry" introduced recently, which in the most basic sense refers to a pair of dual symmetries, such as the Ising symmetries of the $1 d$ quantum Ising model and its self-dual counterpart. In this manuscript we study discrete categorical symmetry at higher dimensional critical points and gapless phases. At these selected gapless states of matter, we can evaluate the behavior of categorical symmetries analytically. We analyze the categorical symmetry at the following examples of criticality: $(i).(2+1) d$ Lifshit critical point of a quantum Ising system; (ii.) $(3+1) d$ photon phase as an intermediate gapless phase between the topological order and the confined phase of $3 d Z_{2}$ quantum gauge theory; (iii.) $2 d$ and $3 d$ examples of systems with both categorical symmetries (either 0-form or 1-form categorical symmetries) and subsystem symmetries. We demonstrate that at some of these gapless states of matter the categorical symmetries have very different behavior from the nearby gapped phases.
\end{abstract}

PACS numbers:

\section{BASICS OF CATEGORICAL SYMMETRY}

Categorical symmetry is a new concept introduced in Ref.1, which expanded the conventional notion of symmetries in physics, and how one should think about them. The basic examples of categorical symmetry correspond to a pair of dual symmetries, whose local symmetry charges in general do not commute with each other. The simplest example of such, are the $Z_{2}$ and $\tilde{Z}_{2}$ dual symmetry of the $1 d$ quantum Ising model:

$$
H=\sum_{j}-K \sigma_{j}^{3} \sigma_{j+1}^{3}-h \sigma_{j}^{1} \quad \leftrightarrow \quad H_{d}=\sum_{\tilde{j}}-K \tau_{\tilde{j}}^{1}-h \tau_{\tilde{j}}^{3} \tau_{\tilde{j}+1}^{3} .
$$

This model has a well-known self-duality point $K=h ; \sigma_{j}^{3}$ and $\tau_{\tilde{j}}^{3}$ are order parameters of the original $Z_{2}$ and the dual $\tilde{Z}_{2}$ symmetry. Let us label the entire categorical symmetries of the $1 d$ quantum Ising model as $Z_{2} \star \tilde{Z}_{2}$.

For the convenience of generalizing to higher dimensional systems with higher form symmetries and more exotic subsystem symmetries that we will discuss in this manuscript, we will introduce the concept "Order Diagnosis Operator" (ODO) for each symmetry. The expectation value of the ODO diagnoses the behavior of its corresponding symmetry. An ODO should commute with all the conserved global symmetry charges (which implies that the expectation value of the ODO is in general nonzerd $\frac{23}{3}$ ), but creates local charges of the corresponding symmetry. For the $Z_{2}$ and $\tilde{Z}_{2}$ symmetries of the $1 d$ quantum Ising model, the ODOs are respectively

$$
O_{i, j}=\sigma_{i}^{3} \sigma_{j}^{3}, \quad \tilde{O}_{\tilde{i}, \tilde{j}}=\tau_{\tilde{i}}^{3} \tau_{\tilde{j}}^{3}=\prod_{i<k<j} \sigma_{k}^{1} .
$$

$O_{i, j}$ creates a pair of $Z_{2}$ charges at sites $i$ and $j$ (but it preserves/commutes with the global $Z_{2}$ charge $\prod_{j} \sigma_{j}^{1}$ ), while $\tilde{O}_{\tilde{i}, \tilde{j}}$ creates a pair of domain walls of $\sigma^{3}$ at $\tilde{i}$ and $\tilde{j}$, which are local charges of the $\tilde{Z}_{2}$ symmetry.

When $K>h$, there is a long range correlation of $\sigma^{3}$, short range correlation of $\tau^{3}$ (long range expectation value of ODO $O_{i, j}$, and short range expectation value of $\left.\tilde{O}_{\tilde{i}, \tilde{j}}\right)$; hence this is a phase that spontaneously breaks $Z_{2}$, but preserves $\tilde{Z}_{2}$. When $K<h$, there is a long range correlation of $\tau^{3}$, but short range correlation of $\sigma^{3}$ (long range expectation value of $\tilde{O}_{\tilde{i}, \tilde{j}}$, short range expectation value of $O_{i, j}$ ); hence this is a phase that spontaneously breaks $\tilde{Z}_{2}$, but preserves $Z_{2}$. Whether a symmetry is preserved or spontaneously broken, can be defined by the behavior of its ODO. When $K=h$, both order parameters have power-law correlation, hence this is a criticality which preserves both symmetries.

In what sense is $\tilde{Z}_{2}$ a symmetry, and in what sense is there a spontaneous symmetry breaking (SSB) of $\tilde{Z}_{2}$ ? In the $1 d$ quantum Ising model, without changing the physical Ising Hilbert space, the SSB phase of the $\tilde{Z}_{2}$ symmetry does not lead to ground state degeneracy (GSD), after all it is just a quantum disordered phase of the Ising model. However, with some global constraint on the physical Hilbert space, or when we view the $1 d$ system as the boundary of a $2 d$ topological order ${ }^{1}$, neither phase $(K>h$ or $K<h)$ has GSD. Hence we no longer view GSD as a criterion for SSB. The SSB should be defined solely by the behavior of $\langle O\rangle$ and $\langle\tilde{O}\rangle$. 
In higher dimensions, the possible categorical symmetries are much richer. In the $2 d$ quantum Ising model, there is a $Z_{2} \star \tilde{Z}_{2}^{(1)}$ symmetry. Here $\tilde{Z}_{2}^{(1)}$ is a 1 -form symmetry as a generalization of ordinary symmetries introduced in recent years (see for instance Ref. $2-10)$ :

$$
\begin{aligned}
H & =\sum_{\left.<\mathbf{x}, \mathbf{x}^{\prime}\right\rangle}-K \sigma_{\mathbf{x}}^{3} \sigma_{\mathbf{x}^{\prime}}^{3}-\sum_{\mathbf{x}} h \sigma_{\mathbf{x}}^{1} \leftrightarrow \\
H_{d} & =\sum_{\tilde{\mathbf{x}}, \hat{\mu}}-K \tau_{\tilde{\mathbf{x}}, \hat{\mu}}^{1}-\sum_{\tilde{\mathbf{x}}} h \tau_{\tilde{\mathbf{x}}, \hat{x}}^{3} \tau_{\tilde{\mathbf{x}}, \hat{y}}^{3} \tau_{\tilde{\mathbf{x}}+\hat{x}, \hat{y}}^{3} \tau_{\tilde{\mathbf{x}}+\hat{y}, \hat{x}}^{3} .
\end{aligned}
$$

The lattice site $\mathbf{x}$ and dual lattice site $\tilde{\mathbf{x}}$ are illustrated in Fig. 1. The subscripts $(\tilde{\mathbf{x}}, \hat{x})$ and $(\tilde{\mathbf{x}}, \hat{y})$ label the links of the dual lattice. The ODO of the $Z_{2}$ symmetry is still $O_{\mathbf{x}, \mathbf{x}^{\prime}}=\sigma_{\mathbf{x}}^{3} \sigma_{\mathbf{x}^{\prime}}^{3}$; while the ODO of $\tilde{Z}_{2}^{(1)}$ symmetry is

$$
\tilde{O}_{\mathcal{C}}^{(1)}=\prod_{\tilde{l} \in \mathcal{C}} \tau_{\tilde{l}}^{3}=\prod_{\mathbf{x} \in \mathcal{A}, \partial \mathcal{A}=\mathcal{C}} \sigma_{\mathbf{x}}^{1}
$$

Here $\tilde{l}$ also labels a link in the dual lattice, which belongs to the contractible loop $\mathcal{C}$. $\tilde{O}_{\mathcal{C}}^{(1)}$ creates an Ising domain wall of $\sigma^{3}$, the one dimensional domain wall carries the dual $\tilde{Z}_{2}^{(1)} 1$-form symmetry charge. Here $\mathcal{A}$ is a finite $2 d$ patch on the dual lattice, $\mathcal{C}$ is the boundary of $\mathcal{A}$, which is a contractible loop. Again, the ODO $\tilde{O}_{\mathcal{C}}^{(1)}$ commutes with all the conserved 1-form symmetry charges, which is defined as a product of $\tau^{1}$ along any closed $1 d$ loop $\mathcal{C}^{\prime}$. Notice that $\mathcal{C}^{\prime}$ always intersects with the contractible $\mathcal{C}$ for even times, hence the ODO $\tilde{O}_{\mathcal{C}}^{(1)}$ commutes with the conserved 1-form symmetry charges $\prod_{\tilde{l} \in \mathcal{C}^{\prime}} \tau_{\tilde{l}}^{1}$.

There are again two phases with $K / h$ greater or smaller than a critical value. These two phases have the following known behaviors of the ODOs ${ }^{11}$, which can be computed through a reliable perturbation theory due to the gap in the spectrum of both phases:

$$
\begin{aligned}
& K / h \gg 1, \quad\left\langle O_{\mathbf{x}, \mathbf{x}^{\prime}}\right\rangle \sim \text { Const, } \quad\left\langle\tilde{O}_{\mathcal{C}}^{(1)}\right\rangle \sim e^{-\alpha_{1} \log (K / h) \mathcal{A}} . \\
& K / h \ll 1, \quad\left\langle O_{\mathbf{x}, \mathbf{x}^{\prime}}\right\rangle \sim e^{-\left|\mathbf{x}-\mathbf{x}^{\prime}\right| / \xi}, \quad\left\langle\tilde{O}_{\mathcal{C}}^{(1)}\right\rangle \sim e^{-\alpha_{2}(K / h)^{2} \mathcal{C}} .
\end{aligned}
$$

$\alpha_{i}$ are order 1 numbers. Hence in the phase $K \gg h$, the $\tilde{Z}_{2}^{(1)}$ symmetry $\tilde{O}_{\mathcal{C}}^{(1)}$ decays with an area law; while in the phase $K \ll h$, the domain walls proliferate/condense, and $\tilde{O}_{\mathcal{C}}^{(1)}$ has a perimeter law. Again, in the phase $h \gg K$, even though the domain walls proliferate/condense, there is no GSD. This is in stark contrast with ordinary 1-form symmetry SSB state, which would lead to topological degeneracy. Hence here we should view the behavior of $\left\langle\tilde{O}_{\mathcal{C}}^{(1)}\right\rangle$ as a criterion of SSB of $\tilde{Z}_{2}^{(1)}$, rather than the GSD.

At the $(2+1) d$ Ising critical point, the $Z_{2}$ order parameter has a power-law correlation (the expectation value of $O_{\mathbf{x}, \mathbf{x}^{\prime}}$ falls off as a power-law), hence the $Z_{2}$ symmetry is not broken. Intuitively, since $O_{\mathbf{x}, \mathbf{x}^{\prime}}$ has a power-law correlation, the expectation value of the dual ODO $\tilde{O}_{\mathcal{C}}^{(1)}$ should be stronger than the area law deep in the $K \gg h$ phase, but weaker than the perimeter law deep in the $K \ll h$ phase. But the exact behavior of $\tilde{O}_{\mathcal{C}}^{(1)}$ is difficult to compute analytically at the $3 D$ Ising critical point, and in other lattice models that will be discussed in the following sections. The main goal of this manuscript is to find critical points (or fine-tuned critical points) where the ODOs of the categorical symmetries can be evaluated analytically. The strategy we will generally take is that, we embed the target lattice model into a larger "parent" system where the ODOs of the original system have a clear representation. Then we tune the parent system to a multi-critical point, or even a gapless phase, where we can use tools in the continuum limit to compute ODOs defined in both sides of the duality. Since many of the states we discuss in this manuscript do not have Lorentz invariance, we will focus on expectation value of time-independent operators at static states.

\section{ISING CATEGORICAL SYMMETRIES AT CRITICALITY}

\section{A. $2 d$ Lifshitz Point}

We can embed the target $2 d$ quantum Ising model into a parent system described by a U(1) quantum "rotor":

$$
H=\sum_{\mathbf{x}, \mu}-t \cos \left(\nabla_{\mu} \hat{\theta}(\mathbf{x})\right)+\sum_{\mathbf{x}} \frac{U}{2} \hat{n}(\mathbf{x})^{2}-g \cos (2 \hat{\theta}(\mathbf{x})) .
$$




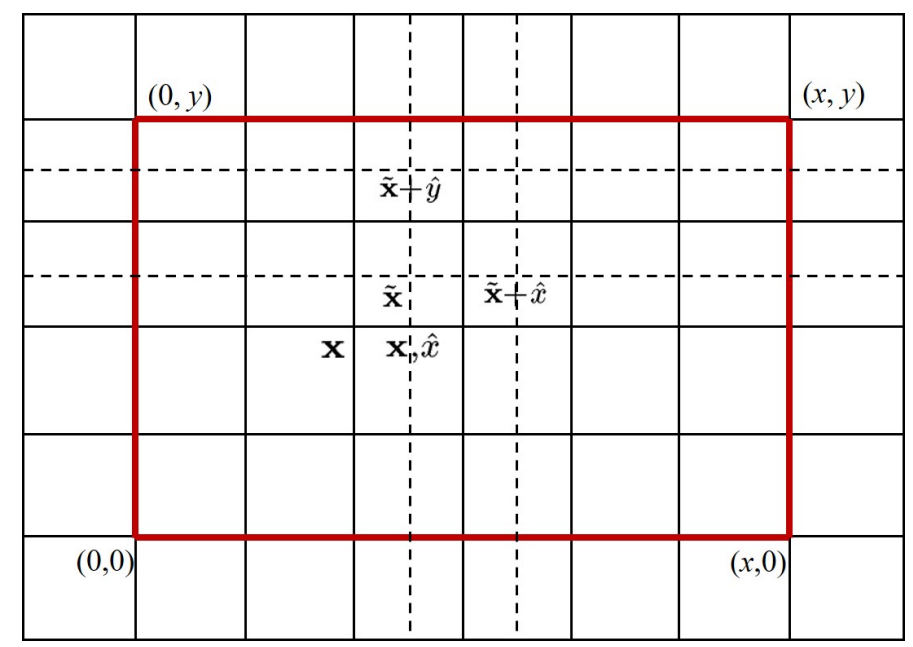

FIG. 1: The $2 d$ square lattice, and its dual lattice. The lattice site is labelled as $\mathbf{x}$, and the dual lattice site (the plaquette of the original lattice) is labelled as $\tilde{\mathbf{x}}$. The links of the lattice are labelled as $(\mathbf{x}, \hat{\mu})$, while the links of the dual lattice are labelled as $(\tilde{\mathbf{x}}, \hat{\mu})$.

$\hat{\theta}(\mathbf{x})$ and $\hat{n}(\mathbf{x})$ are a pair of conjugate variables, i.e. $\left[\hat{n}(\mathbf{x}), \hat{\theta}\left(\mathbf{x}^{\prime}\right)\right]=\mathrm{i} \delta_{\mathbf{x}, \mathbf{x}^{\prime}} \cdot \hat{n}(\mathbf{x})$ takes discrete integer eigenvalues, while $\hat{\theta}(\mathbf{x})$ is periodically defined: $\hat{\theta}(\mathbf{x})=\hat{\theta}(\mathbf{x})+2 \pi$. The last $g$ term in Eq. 6 breaks the U(1) symmetry down to $Z_{2}$. The operators $\sigma_{\mathbf{x}}^{3}$ and $\sigma_{\mathbf{x}}^{1}$ of the Ising model correspond to the operators in the parent $\mathrm{U}(1)$ theory:

$$
\sigma_{\mathbf{x}}^{3}=e^{\mathrm{i} \hat{\theta}(\mathbf{x})}, \quad \sigma_{\mathbf{x}}^{1}=e^{\mathrm{i} \pi \hat{n}(\mathbf{x})} .
$$

If the $g$ term is ignored, the $\mathrm{U}(1)$ model is dual to a lattice QED:

$$
\begin{aligned}
& H_{d}=\sum_{\tilde{\mathbf{x}}}-t \cos (\hat{\vec{e}}(\tilde{\mathbf{x}}))+\sum_{\tilde{\mathbf{x}}} \frac{U}{2}(\vec{\nabla} \times \hat{\vec{a}}(\tilde{\mathbf{x}}))^{2} \\
& \hat{\vec{e}}(\tilde{\mathbf{x}})=\hat{z} \times \vec{\nabla} \hat{\theta}(\mathbf{x}), \quad \vec{\nabla} \times \hat{\vec{a}}(\tilde{\mathbf{x}})=\hat{n}(\mathbf{x}) .
\end{aligned}
$$

The electric field $\hat{e}_{\mu}$ and gauge vector potential $\hat{a}_{\mu}$ were defined on the links $(\tilde{\mathbf{x}}, \hat{x}),(\tilde{\mathbf{x}}, \hat{y})$ of the dual lattice, but we can also equivalently define $\hat{\vec{e}}(\tilde{\mathbf{x}})=\left(\hat{e}_{x}(\tilde{\mathbf{x}}), \hat{e}_{y}(\tilde{\mathbf{x}})\right)=\left(\hat{e}_{\tilde{\mathbf{x}}}, \hat{x}, \hat{e}_{\tilde{\mathbf{x}}, \hat{y}}\right), \hat{\vec{a}}(\tilde{\mathbf{x}})=\left(\hat{a}_{x}(\tilde{\mathbf{x}}), \hat{a}_{y}(\tilde{\mathbf{x}})\right)=\left(\hat{a}_{\tilde{\mathbf{x}}, \hat{x}}, \hat{a}_{\tilde{\mathbf{x}}, \hat{y}}\right)$. In the parent $\mathrm{U}(1)$ system, the $Z_{2}$ and $\tilde{Z}_{2}^{(1)}$ ODO are

$$
\begin{aligned}
& O_{\mathbf{x}, \mathbf{x}^{\prime}}=e^{\mathrm{i} \hat{\theta}(\mathbf{x})} e^{-\mathrm{i} \hat{\theta}\left(\mathbf{x}^{\prime}\right)} \\
& \tilde{O}_{\mathcal{C}}^{(1)}=\prod_{\mathcal{A}, \partial \mathcal{A}=\mathcal{C}} \sigma_{\mathbf{x}}^{1}=\exp \left(i \pi \sum_{\mathbf{x} \in \mathcal{A}} \hat{n}(\mathbf{x})\right)=\exp \left(i \pi \oint_{\mathcal{C}} \hat{\vec{a}} \cdot d \vec{l}\right) .
\end{aligned}
$$

In model Eq. 6, there is a critical point at critical value $(U / t)_{c}$. Without the $g$ term, the transition in Eq. 6 is a $3 D \mathrm{XY}$ transition between the superfluid phase with small $U / t$ and a boson Mott insulator phase at large $U / t$. While with the $g$ term, it is expected that the $3 D \mathrm{XY}$ critical point will flow to the $3 D$ Ising fixed point, because $g$ is obviously relevant at the $3 D \mathrm{XY}$ fixed point. However, one can fine-tune the critical point to reach a Lifshitz point described by the following field theory Hamiltonian and action in the continuum limit

$$
\begin{aligned}
& H=\int d^{2} x \frac{U}{2} \hat{n}(\mathbf{x})^{2}+\frac{\rho}{2}\left(\nabla^{2} \hat{\theta}(\mathbf{x})\right)^{2}, \\
& \mathcal{S}=\int d^{2} x d \tau \frac{1}{2 U}\left(\partial_{\tau} \theta\right)^{2}+\frac{\rho}{2}\left(\nabla^{2} \theta\right)^{2} .
\end{aligned}
$$

It is known that the $g$ operator can be irrelevant at the $(2+1) d$ Lifshitz Gaussian fixed point for certain range of $U$ and $\rho$, more precisely for large enough $U / \rho^{12 \mid 13}$. The irrelevance of $g$ guarantees that the continuum limit field theory description in terms of $\theta$ is applicable at this Lifshitz fixed point. One can also compute the expectation value of $O$, 
which is the equal-time correlation function between $\sigma^{3}$ :

$$
\left\langle O_{0, \mathbf{x}}\right\rangle=\left\langle e^{\mathrm{i} \hat{\theta}(0)} e^{-\mathrm{i} \hat{\theta}(\mathbf{x})}\right\rangle \sim \frac{1}{|\mathbf{x}|^{2 \Delta_{\theta}}}, \quad \Delta_{\theta} \sim \sqrt{\frac{U}{\rho}} .
$$

Hence at the Lifshitz point, the $Z_{2}$ symmetry is preserved.

The situation is rather different for the $Z_{2}^{(1)}$ ODO $\tilde{O}_{\mathcal{C}}$. The dual Hamiltonian and action of the Lifshitz theory Eq. 10 is

$$
\begin{aligned}
& H_{d}=\int d^{2} \tilde{x} \frac{U}{2}(\vec{\nabla} \times \hat{\vec{a}})^{2}+\frac{\rho}{2}\left(\left(\nabla_{x} \hat{e}_{y}\right)^{2}+\left(\nabla_{y} \hat{e}_{x}\right)^{2}\right), \\
& \mathcal{S}_{d}=\int d^{2} \tilde{x} d \tau \frac{1}{2 \rho}\left(\hat{a}_{x} \frac{\partial_{\tau}^{2}}{\partial_{y}^{2}} \hat{a}_{x}+\hat{a}_{y} \frac{\partial_{\tau}^{2}}{\partial_{x}^{2}} \hat{a}_{y}\right)+\frac{U}{2}(\vec{\nabla} \times \hat{\vec{a}})^{2} .
\end{aligned}
$$

This is the same Hamiltonian and action describing the $2 d$ quantum dimer model at the Rohksar-Kivelson point 1415 . The correlation function of $\vec{a}_{\vec{q}, \omega}$ is

$$
\left\langle\hat{a}_{\mu}(-\omega,-\vec{q}) \hat{a}_{\nu}(\omega, \vec{q})\right\rangle \sim \frac{\rho\left(q^{2} \delta_{\mu \nu}-q_{\mu} q_{\nu}\right)}{\omega^{2}+\rho U q^{4}}, \quad\left\langle\hat{a}_{\mu}(0,0) \hat{a}_{\nu}(0, \mathbf{x})\right\rangle \sim \sqrt{\frac{\rho}{U}} \frac{1}{|\mathbf{x}|^{2}}
$$

The expectation of $\tilde{O}_{\mathcal{C}}^{(1)}$ can be evaluated using the Gaussian theory of the gauge field:

$$
\left\langle\exp \left(\mathrm{i} \pi \oint_{\mathcal{C}} \hat{\vec{a}} \cdot d \vec{l}\right)\right\rangle \sim \exp \left(-\frac{\pi^{2}}{2} \oint_{\mathcal{C}} \oint_{\mathcal{C}}\left\langle\hat{a}_{\mu}(\mathbf{x}) \hat{a}_{\nu}\left(\mathbf{x}^{\prime}\right)\right\rangle d x^{\mu} d x^{\prime \nu}\right)
$$

Power-counting suggests that this is still a perimeter law: the $1 /|\mathbf{x}|^{2}$ decay of the correlation function of the gauge fields do not lead to extra divergence with large loop size, the expectation value of $\tilde{O}_{\mathcal{C}}^{(1)}$ is dominated by small distance correlation of the gauge field. Since in the gapped phase $h \gg K$ (Eq. 3) where the domain walls clearly proliferates,

\begin{tabular}{|c|c|c|c|}
\hline $2 d$ Quantum Ising theory & $K \gg h$ in Eq. 3 & $K \ll h$ in Eq. $\sqrt{3}$ & Fine-tuned Lifshitz Point \\
\hline$O_{\mathbf{x}, \mathbf{x}^{\prime}}$ & Long range & Short Range & Power law \\
\hline$\tilde{O}_{\mathcal{C}}^{(1)}$ & Area law & Perimeter law & Perimeter law \\
\hline
\end{tabular}
$\tilde{O}_{\mathcal{C}}^{(1)}$ follows a perimeter law, we will use the perimeter law of $\tilde{O}_{\mathcal{C}}^{(1)}$ as a criterion of SSB of $\tilde{Z}_{2}^{(1)}$. Then this Lifshitz point still spontaneously breaks the $\tilde{Z}_{2}^{(1)}$ symmetry, while preserving the $Z_{2}$ symmetry. One can also see that when the expectation value of $O_{\mathbf{x}, \mathbf{x}^{\prime}}$ is stronger $\left(\right.$ smaller $\Delta_{\theta}$ at smaller $\left.U / \rho\right)$, the expectation value of $\tilde{O}_{\mathcal{C}}^{(1)}$ becomes weaker (larger $\rho / U)$. The results of this section are summarized in the table below.

\section{B. 3d $Z_{2}$ Quantum Gauge Theory}

It was well-known that the $3 d$ lattice $Z_{2}$ gauge theory has a self-dual structure $11|16| 17$ :

$$
\begin{aligned}
H & =\sum_{\mathbf{x}, \hat{\mu}, \hat{\nu}}-K \sigma_{\mathbf{x}, \hat{\mu}}^{3} \sigma_{\mathbf{x}, \hat{\nu}}^{3} \sigma_{\mathbf{x}+\hat{\mu}, \hat{\nu}}^{3} \sigma_{\mathbf{x}+\hat{\nu}, \hat{\mu}}^{3}-h \sigma_{\mathbf{x}, \hat{\mu}}^{1} \\
\leftrightarrow \quad H_{d} & =\sum_{\tilde{\mathbf{x}}, \hat{\mu}, \hat{\nu}}-K \tau_{\tilde{\mathbf{x}}, \hat{\mu}}^{1}-h \tau_{\tilde{\mathbf{x}}, \hat{\mu}}^{3} \tau_{\tilde{\mathbf{x}}, \hat{\nu}}^{3} \tau_{\tilde{\mathbf{x}}+\hat{\mu}, \hat{\nu}}^{3} \tau_{\tilde{\mathbf{x}}+\hat{\nu}, \hat{\mu}}^{3}
\end{aligned}
$$

This system has a $Z_{2}^{(1)} \star \tilde{Z}_{2}^{(1)}$ categorical symmetry. The ODOs for $Z_{2}^{(1)}$ and $\tilde{Z}_{2}^{(1)}$ are

$$
O_{\mathcal{C}}^{(1)}=\prod_{l \in \mathcal{C}} \sigma_{l}^{3}, \quad \tilde{O}_{\mathcal{C}}^{(1)}=\prod_{\tilde{l} \in \mathcal{C}} \tau_{\tilde{l}}^{3}
$$

The $O_{\mathcal{C}}^{(1)}$ and $\tilde{O}_{\mathcal{C}}^{(1)}$ are products of the $K$ and $h$ terms of Eq. 15 within $2 d$ patch $\mathcal{A}$ with $\partial \mathcal{A}=\mathcal{C}$.

There are two phases of this model: for $K \gg h,\left\langle O_{\mathcal{C}}^{(1)}\right\rangle$ decays with a perimeter law, while $\left\langle\tilde{O}_{\mathcal{C}}^{(1)}\right\rangle$ decays with an area law; this is a phase with SSB of $Z_{2}^{(1)}$ but preserves $\tilde{Z}_{2}^{(1)}$. 
In the opposite limit $h \gg K,\left\langle O_{\mathcal{C}}^{(1)}\right\rangle$ decays with an area law, while $\left\langle\tilde{O}_{\mathcal{C}}^{(1)}\right\rangle$ decays with an perimeter law; this is the phase with SSB of $\tilde{Z}_{2}^{(1)}$ but preserves $Z_{2}^{(1)}$.

Unfortunately, model Eq. 15 does not have a second order transition between the two phases, hence there is no critical point in model Eq. 15 where $Z_{2}^{(1)}$ and $\tilde{Z}_{2}^{(1)}$ are on equal footing. But we can embed the $Z_{2}$ gauge theory Eq. 15 into a QED model with $U(1)^{(1)} \star \tilde{U}(1)^{(1)}$ symmetries, and this QED model has a gapless photon phase. In this gapless photon phase, both $O_{\mathcal{C}}^{(1)}$ and $O_{\mathcal{C}^{\prime}}^{(1)}$ in Eq. 16 can be computed using the Gaussian fixed point theory of the $U(1)$ gauge field, and its self-dual $\tilde{U}(1)$ gauge field. The Gaussian theory of the $U(1)$ and $\tilde{U}(1)$ gauge bosons indicates that both $O_{\mathcal{C}}$ and $\tilde{O}_{\mathcal{C}}$ follow a perimeter law. Since in the gapped phases of Eq. $15 O_{\mathcal{C}}$ and $\tilde{O}_{\mathcal{C}}$ at most have a perimeter law, we view the gapless photon phase of the $U(1)$ gauge field as a phase which spontaneously breaks both $Z_{2}^{(1)}$ and $\tilde{Z}_{2}^{(1)}$ symmetries. This gapless QED would still have $Z_{2}^{(1)} \star \tilde{Z}_{2}^{(1)}$ as the UV symmetry, while the $U(1)^{(1)} \star \tilde{U}(1)^{(1)}$ symmetry are IR emergent symmetries. The IR emergent symmetries are spontaneously broken, which still leads to gapless photons as their Goldstone modes 24 .

One can also fine-tune the QED to a Lifshitz point with non-Lorentz invariant dispersions of the $U(1)$ gauge bosons. However, we have checked and verified that, at various Lifshitz points (meaning fine-tuned states with different nonLorentz invariant dispersion), at least one of the $Z_{2}^{(1)}$ and $\tilde{Z}_{2}^{(1)}$ symmetries is spontaneously broken, i.e. one of $O_{\mathcal{C}}$ and $\tilde{O}_{\mathcal{C}}$ must have a perimeter law.

\section{EXAMPLES OF SUBSYSTEM CATEGORICAL SYMMETRIES}

\section{A. 2d Example}

Let us consider a special $2 d$ lattice $Z_{2}$ quantum gauge theory, which can be constructed in Josephson arrays of superconductor and ferromagnet deposited on top of a quantum spin Hall insulator 18 :

$$
H=\sum_{\mathbf{x}}-K \sigma_{\mathbf{x}, \hat{x}}^{3} \sigma_{\mathbf{x}, \hat{y}}^{3} \sigma_{\mathbf{x}+\hat{x}, \hat{y}}^{3} \sigma_{\mathbf{x}+\hat{y}, \hat{x}}^{3}-J \sigma_{\mathbf{x}, \hat{x}}^{1} \sigma_{\mathbf{x}+\hat{x}, \hat{x}}^{1}-J \sigma_{\mathbf{x}, \hat{y}}^{1} \sigma_{\mathbf{x}+\hat{y}, \hat{y}}^{1}
$$

The last two terms of this model are actually identical, due to the $Z_{2}$ Gauss law gauge constraint $\sigma_{\mathbf{x}-\hat{x}, \hat{x}}^{1} \sigma_{\mathbf{x}, \hat{x}}^{1} \sigma_{\mathbf{x}-\hat{y}, \hat{y}}^{1} \sigma_{\mathbf{x}, \hat{y}}^{1}=1$, which we will impose strictly on the Hilbert space of the system.

This model has an ordinary $Z_{2}^{(1)} 1$-form symmetry, and extra $Z_{2}^{(\mathrm{sub})}$ subsystem symmetries. The subsystem symmetry grants the system a series of conserved quantities:

$$
\Sigma_{\hat{x}, y}=\prod_{y=\text { Const }} \sigma_{\mathbf{x}, \hat{x}}^{3}, \quad \Sigma_{\hat{y}, x}=\prod_{x=\text { Const }} \sigma_{\mathbf{x}, \hat{y}}^{3} .
$$

$x$ and $y$ are the two coordinates of $\mathbf{x}$. The subsystem symmetries of Eq. 17 guarantee that $\Sigma_{\hat{x}, y}$ and $\Sigma_{\hat{y}, x}$ are conserved for arbitrary $x$ and $y$. The ODO for $Z_{2}^{(1)}$, and its expectation value in the topological ordered phase $K \gg J$ is

$$
O_{\mathcal{C}}^{(1)}=\prod_{l \in \mathcal{C}} \sigma_{l}^{3}, \quad\left\langle O_{\mathcal{C}}^{(1)}\right\rangle \sim e^{-\alpha_{3}(J / K)^{2} N_{\mathcal{C}}} .
$$

The $O_{\mathcal{C}}^{(1)}$ commutes with conserved quantities $\Sigma_{\hat{x}, y}$ and $\Sigma_{\hat{y}, x}$, hence it meets the criterion of ODO we introduced in the first section. Due to the conservation of the extra quantities $\Sigma_{\hat{x}, y}$ and $\Sigma_{\hat{y}, x}$, the ODO has a generic "corner law" instead of perimeter law, where $N_{\mathcal{C}}$ is the number of corners of loop $\mathcal{C}$. For example, in Fig. 1. the rectangular loop $\mathcal{C}$ has four corners, And $O_{\mathcal{C}}^{(1)}$ is a product of finite segments of $\Sigma_{\hat{x}, y}$ and $\Sigma_{\hat{y}, x}$. The expectation value of the rectangular $O_{\mathcal{C}}^{(1)}$ does not decay with the length of $\mathcal{C}$. Because $\Sigma_{\hat{x}, y}$ and $\Sigma_{\hat{y}, x}$ are conserved when the product is along an infinitely straight line, then for a generic $\mathcal{C}$, if we compute the expectation value of $O_{\mathcal{C}}^{(1)}$ through a perturbation of $J / K$ like Ref. 11, the value can only decay when $\mathcal{C}$ "takes a turn".

In the other limit of the model, $K \ll J$, the ODO $O_{\mathcal{C}}^{(1)}$ decays as an area law like the ordinary confined phase of a $Z_{2}$ lattice gauge theory, and there is a SSB of the subsystem symmetries $Z_{2}^{(\mathrm{sub})}$. The most convenient way to study this limit, is to take the dual Hamiltonian of Eq. 17. which still has subsystem $\tilde{Z}_{2}^{(\mathrm{sub})}$ symmetries:

$$
H_{d}=\sum_{\tilde{\mathbf{x}}}-K \tau_{\tilde{\mathbf{x}}}^{1}-2 J \tau_{\tilde{\mathbf{x}}}^{3} \tau_{\tilde{\mathbf{x}}+\hat{x}}^{3} \tau_{\tilde{\mathbf{x}}+\hat{y}}^{3} \tau_{\tilde{\mathbf{x}}+\hat{x}+\hat{y}}^{3} .
$$


The duality mapping between $\sigma^{i}$ and $\tau^{i}$ is the same as the standard $2 d$ Ising-Gauge duality discussed in the previous section. $\tilde{Z}_{2}^{(\text {sub) }}$ inherits and contains $Z_{2}^{(\text {sub) }}$, but is slightly larger: $\tilde{Z}_{2}^{\text {(sub) }}$ includes another $\tilde{Z}_{2}$ element which changes the sign of all $\tau_{\tilde{\mathbf{x}}}^{3}$. This extra $\tilde{Z}_{2}$ element is the dual of $Z_{2}^{(1)}$, and it does not change $\sigma_{l}^{1}$ in Eq. 17 .

The ODO of $\tilde{Z}_{2}^{(\mathrm{sub})}$ is a product of $\tau^{3}$ on four corners of a rectangle:

$$
\tilde{O}_{x, y}^{(\mathrm{sub})}=\tau_{0,0}^{3} \tau_{x, 0}^{3} \tau_{0, y}^{3} \tau_{x, y}^{3} .
$$

The ODO defined above is also a product of the $J$ term in Eq. 17 within the rectangle. In the original topological order $K \gg J, \tilde{O}_{x, y}^{(\mathrm{sub})}$ can be computed through a perturbation of $J / K$, and it decays as an exponential of the area of the rectangle; while at the SSB phase of $\tilde{Z}_{2}^{(\mathrm{sub})}(K \ll J), \tilde{O}_{x, y}^{(\mathrm{sub})}$ has long range expectation value ${ }^{[19}$.

Like the previous section, we can embed the dual model Eq. 20 into a model with $\tilde{U}(1)^{(\text {sub })}$ symmetry:

$$
H_{d}=\int d^{2} \tilde{x} \frac{U}{2} \hat{n}(\tilde{\mathbf{x}})^{2}-t \cos \left(\nabla_{x} \nabla_{y} \hat{\theta}(\tilde{\mathbf{x}})\right)-g \cos (2 \hat{\theta}(\tilde{\mathbf{x}})) .
$$

The relation between the operators of the $\tilde{Z}_{2}^{(\mathrm{sub})}$ theory Eq. 20 and the $\tilde{U}(1)^{(\mathrm{sub})}$ theory Eq. 22 is

$$
\tau_{\tilde{\mathbf{x}}}^{x}=\exp (\mathrm{i} \pi \hat{n}(\tilde{\mathbf{x}})), \quad \tau_{\tilde{\mathbf{x}}}^{z}=\exp (\mathrm{i} \hat{\theta}(\tilde{\mathbf{x}}))
$$

When $g$ is relevant, it will break the $\tilde{U}(1)^{(\mathrm{sub})}$ down to $\tilde{Z}_{2}^{(\mathrm{sub})}$.

However, as was studied before 20 , the $g$ term can only flow strong and become nonperturbative under renormalization group through "assistance" from some other terms such as $\gamma\left(2 \nabla_{\mu} \theta\right)$. If we tune $\gamma$ to zero, then there exists a stable gapless phase of the model Eq. 22 with a larger $\tilde{U}(1)^{(\mathrm{sub})}$ symmetry, and the $g$ term is irrelevant. And in this gapless phase the system is described by the following action:

$$
\mathcal{S}_{d}=\int d \tau d^{2} \tilde{x} \frac{1}{2 U}\left(\partial_{\tau} \theta\right)^{2}+\frac{t}{2}\left(\nabla_{x} \nabla_{y} \theta\right)^{2},
$$

where $\theta$ can be viewed as a free boson instead of a compact boson. The $\tilde{U}(1)^{(\mathrm{sub})}$ reads

$$
\theta(\tilde{\mathbf{x}}) \rightarrow \theta(\tilde{\mathbf{x}})+f(\tilde{x})+g(\tilde{y}) .
$$

This gapless phase can also be described by a $U(1)$ gauge theory, which can be viewed as the parent theory where the original $Z_{2}$ lattice gauge theory Eq. 17 is embedded to:

$$
H=\int d^{2} x \frac{U}{2}(\vec{\nabla} \times \hat{\vec{a}})^{2}+\frac{t}{4}\left(\left(\nabla_{x} \hat{e}_{x}\right)^{2}+\left(\nabla_{y} \hat{e}_{y}\right)^{2}\right) .
$$

In this gapless phase, the expectation value of the ODO of the original $Z_{2}$ gauge theory $O_{\mathcal{C}}^{(1)}$ will depend on the shape of $\mathcal{C}$, but it no longer follows the "corner law" Eq. 19 of the gapped topological ordered phase $K \gg J$ in Eq. 17 . In the gapless phase, the ODO $O_{\mathcal{C}}^{(1)}$ can be written as

$$
\left\langle O_{\mathcal{C}}^{(1)}\right\rangle=\left\langle\prod_{\tilde{\mathbf{x}} \in \mathcal{A}, \partial \mathcal{A}=\mathcal{C}} \tau_{\tilde{\mathbf{x}}}^{1}\right\rangle \sim\left\langle e^{\sum_{\tilde{\mathbf{x}} \in \mathcal{A}} \mathrm{i} \pi \hat{n}(\tilde{\mathbf{x}})}\right\rangle
$$

In order to evaluate $\left\langle O_{\mathcal{C}}^{(1)}\right\rangle$ we will make use of another duality of Eq. 22 and Eq. 24

$$
H_{d 2}=\int d^{2} x \frac{U}{2}\left(\nabla_{x} \nabla_{y} \hat{\phi}(\mathbf{x})\right)^{2}-t \cos (\hat{N}(\mathbf{x})) .
$$

Now $\hat{\phi}(\mathbf{x})$ and $\hat{N}(\mathbf{x})$ are still defined on the sites of the original lattice $\mathbf{x}$ (Fig. 11):

$$
\nabla_{x} \nabla_{y} \hat{\theta}(\tilde{\mathbf{x}})=-\hat{N}(\mathbf{x}), \quad \nabla_{x} \nabla_{y} \hat{\phi}(\mathbf{x})=\hat{n}(\tilde{\mathbf{x}}) .
$$

The gapless phase has a new dual description in terms of the continuum limit model of $\hat{\phi}(\mathbf{x})$ :

$$
\mathcal{S}_{2 d}=\int d^{2} x d \tau \frac{1}{2 t}\left(\partial_{\tau} \phi\right)^{2}+\frac{U}{2}\left(\nabla_{x} \nabla_{y} \phi\right)^{2} .
$$




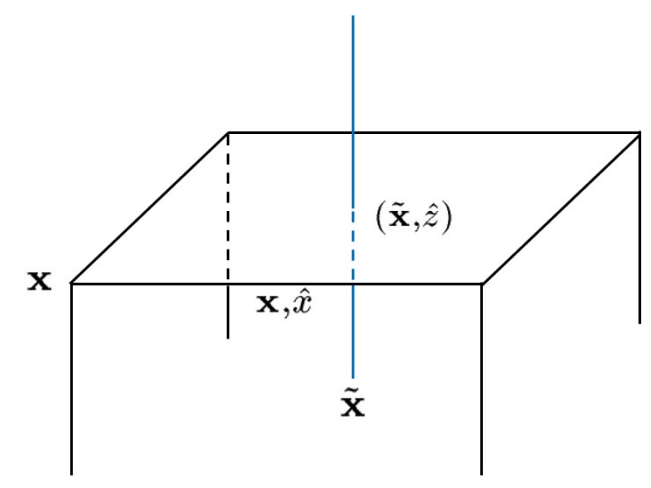

FIG. 2: The cubic lattice and the dual lattice for models considered in section IIIB.

In this gapless phase, if we consider a loop $\mathcal{C}$ which is a rectangle with four corners at $(0,0),(x, 0),(0, y),(x, y)$ (Fig. 1), the expectation value $O_{\mathcal{C}}^{(1)}$ is

$$
\begin{aligned}
& \left\langle O_{\mathcal{C}}^{(1)}\right\rangle=\left\langle\prod_{\tilde{\mathbf{x}} \in \mathcal{A}, \partial \mathcal{A}=\mathcal{C}} \tau_{\tilde{\mathbf{x}}}^{1}\right\rangle \sim\left\langle\exp \left(\sum_{\tilde{\mathbf{x}} \in \mathcal{A}} \mathrm{i} \pi \hat{n}(\tilde{\mathbf{x}})\right)\right\rangle \\
= & \left\langle\exp \left(\mathrm{i} \pi\left(\hat{\phi}_{0,0}-\hat{\phi}_{x, 0}-\hat{\phi}_{0, y}+\hat{\phi}_{x, y}\right)\right)\right\rangle \\
\sim & \exp \left(\pi^{2}\left(\left\langle\hat{\phi}_{0,0} \hat{\phi}_{x, 0}\right\rangle+\left\langle\hat{\phi}_{0,0} \hat{\phi}_{0, y}\right\rangle+\left\langle\hat{\phi}_{x, y} \hat{\phi}_{x, 0}\right\rangle+\left\langle\hat{\phi}_{x, y} \hat{\phi}_{0, y}\right\rangle-\left\langle\hat{\phi}_{0,0} \hat{\phi}_{x, y}\right\rangle-\left\langle\hat{\phi}_{0, y} \hat{\phi}_{x, 0}\right\rangle\right)\right) \\
\sim & \exp \left(-c \pi^{2} \sqrt{\frac{t}{U}} \log |x| \log |y|\right) .
\end{aligned}
$$

This is a faster decay compared with the corner law in the gapped topologically ordered phase $K \gg J$ in Eq. 17. In the same gapless phase, the expectation value of $\tilde{O}_{x, y}^{\text {sub }}$ defined in Eq. 21 decays in a similar way as Eq. 31 rather than a long range expectation value as the phase $K \ll J$. Hence this gapless phase described by Eq. 24. Eq. 26 , Eq. 30 can be viewed as a symmetric phase for both $Z_{2}^{(1)}$ and $\tilde{Z}_{2}^{\text {sub }}$ symmetries.

The special double logarithmic scaling in Eq. 31 arises from the subsystem symmetries Eq. 25 of the parent $U(1)$ theory. More technically, in order to evaluate $O_{\mathcal{C}}^{(1)}$, we need to compute the equal-time correlation function $\left\langle\hat{\phi}_{0,0} \hat{\phi}_{x, y}\right\rangle$,

\begin{tabular}{|c|c|c|c|}
\hline Special $2 d Z_{2}$ Gauge theory Eq. 17 & $K \gg J$ & $K \ll J$ & Gapless Phase \\
\hline$O_{\mathcal{C}}^{(1)}$ & Corner law & Area law & $\exp \left(-c \pi^{2} \sqrt{t / U} \log |x| \log |y|\right)$ for rect. $\mathcal{C}$ \\
\hline$\tilde{O}_{x, y}^{\text {sub }}$ & Area law & Long range & $\exp \left(-\tilde{c} \pi^{2} \sqrt{U / t} \log |x| \log |y|\right)$ \\
\hline
\end{tabular}
which in the momentum space is ${ }^{20} G_{k_{x}, k_{y}} \sim \int d \omega \omega t /\left(\omega^{2}+t U k_{x}^{2} k_{y}^{2}\right) \sim 1 /\left|k_{x} k_{y}\right|$. The double linear divergence at $k_{x} \rightarrow 0$ and $k_{y} \rightarrow 0$ leads to the special double logarithmic scaling in real space. The results of this subsection is summarized in the table below.

\section{B. 3d Example}

We now consider a $3 d Z_{2}$ lattice gauge theory defined on the cubic lattice, which has both the 1-form symmetry, and subsystem symmetries:

$$
\begin{aligned}
H & =\sum_{\mathbf{x}, \hat{\mu}, \hat{\nu}}-K \sigma_{\mathbf{x}, \hat{\mu}}^{3} \sigma_{\mathbf{x}, \hat{\nu}}^{3} \sigma_{\mathbf{x}+\hat{\mu}, \hat{\nu}}^{3} \sigma_{\mathbf{x}+\hat{\nu}, \hat{\mu}}^{3}-J \sigma_{\mathbf{x}, \hat{\mu}}^{1} \sigma_{\mathbf{x}+\hat{\mu}, \hat{\mu}}^{1} \\
\leftrightarrow \quad H_{d} & =\sum_{\tilde{\mathbf{x}}, \hat{\mu}, \hat{\nu}}-K \tau_{\tilde{\mathbf{x}}, \hat{\mu}}^{1}-\sum_{\hat{\rho} \perp \hat{\mu}, \hat{\nu}} J \hat{\mathcal{B}}_{\tilde{\mathbf{x}}, \hat{\mu} \hat{\nu}} \hat{\mathcal{B}}_{\tilde{\mathbf{x}}+\hat{\rho}, \hat{\mu} \hat{\nu}}
\end{aligned}
$$

where $\hat{\mathcal{B}}_{\tilde{\mathbf{x}}, \hat{\mu} \hat{\nu}}=\tau_{\tilde{\mathbf{x}}, \hat{\mu}}^{3} \tau_{\tilde{\mathbf{x}}, \hat{\nu}}^{3} \tau_{\tilde{\mathbf{x}}+\hat{\mu}, \hat{\nu}}^{3} \tau_{\tilde{\mathbf{x}}+\hat{\nu}, \hat{\mu}}^{3}$. The theory $H$ has an ordinary $Z_{2}^{(1)}$ symmetry like Eq. 15 , plus subsystem symmetries with conserved quantities:

$$
\Sigma_{\hat{x} ;(y, z)}=\prod_{y, z=\text { Const }} \sigma_{\mathbf{x}, \hat{x}}^{3}, \quad \Sigma_{\hat{y} ;(x, z)}=\prod_{x, z=\text { Const }} \sigma_{\mathbf{x}, \hat{y}}^{3}, \quad \Sigma_{\hat{z} ;(x, y)}=\prod_{x, y=\text { Const }} \sigma_{\mathbf{x}, \hat{z}}^{3} .
$$


$x, y, z$ are the three coordinates of $\mathbf{x}$. The ODO of the $Z_{2}^{(1)} 1$-form symmetry is the same as Eq. $15 . O_{\mathcal{C}}^{(1)}=\prod_{l \in \mathcal{C}} \sigma_{l}^{3}$. Due to the extra subsystem conserved quantities in Eq. 33 and since $O_{\mathcal{C}}^{(1)}$ is a product of segments of these extra conserved quantities, the expectation value of $O_{\mathcal{C}}^{(1)}$ in the phase $K \gg J$ also decays with a corner law, i.e. the expectation value of $O_{\mathcal{C}}^{(1)}$ decays only when $\mathcal{C}$ takes a turn; in the phase $K \ll J$, there is a SSB of the subsystem symmetry, and the expectation value of $O_{\mathcal{C}}^{(1)}$ decays with an area law.

The dual Hamiltonian $H_{d}$ has the same $\tilde{Z}_{2}^{(1)}$ symmetry as the dual of the ordinary $Z_{2}$ quantum lattice gauge theory, with extra subsystem symmetries as well. The ODO we will consider for $H_{d}$ is

$$
\tilde{O}_{\mathcal{C}, \mathcal{C}^{\prime}}^{(1)}=\prod_{\tilde{l} \in \mathcal{C}} \tau_{\tilde{l}}^{3} \prod_{\tilde{l} \in \mathcal{C}^{\prime}} \tau_{\tilde{l}}^{3}
$$

There are still subsystem symmetries in $H_{d}$ of Eq. 32, with conserved subsystem symmetry charges such as

$$
\tilde{\Sigma}_{\hat{z} ;(\tilde{y}, \tilde{z})}=\prod_{\tilde{y}, \tilde{z}=\text { Const }} \tau_{\tilde{\mathbf{x}}, \hat{z}}^{1}, \quad \tilde{\Sigma}_{\hat{z} ;(\tilde{x}, \tilde{z})}=\prod_{\tilde{x}, \tilde{z}=\text { Const }} \tau_{\tilde{\mathbf{x}}, \hat{z}}^{1}, \quad \cdots
$$

These conserved subsystem charges are not entirely independent from each other due to the Gauss-law gauge constraint imposed on $\tau^{1}$. Due to these subsystem symmetries in the dual model, we restrict our discussions to the cases when $\mathcal{C}$ and $\mathcal{C}^{\prime}$ in $\tilde{O}_{\mathcal{C}, \mathcal{C}^{\prime}}^{(1)}$ are completely parallel with each other, and separated along the direction orthogonal to both loops, (for example, $\mathcal{C}$ and $\mathcal{C}^{\prime}$ can be identical squares in two XY planes, but separated along the $\hat{z}$ direction), because only then would the ODO commute with all the conserved 1-form charges of the dual model Eq. 15 , and also commute with the subsystem conserved charges $\tilde{\Sigma}$. When $\mathcal{C}$ and $\mathcal{C}^{\prime}$ are identical loops in XY planes separated along the $\hat{z}$ direction, $\tilde{O}_{\mathcal{C}, \mathcal{C}^{\prime}}^{(1)}$ is also a product of $J \sigma_{\mathbf{x}, \hat{z}}^{1} \sigma_{\mathbf{x}+\hat{z}, \hat{z}}^{1}$ in $H$ of Eq. 32 within the $3 d$ region sandwiched between $\mathcal{C}$ and $\mathcal{C}^{\prime}$; while $O_{\mathcal{C}}^{(1)}$ is still a product of the $K$ term enclosed by $\mathcal{C}$.

In the phase $K \ll J$, the expectation value of $\tilde{O}_{\mathcal{C}, \mathcal{C}^{\prime}}^{(1)}$ can again be computed through a perturbation of $K / J$ : it decays as a perimeter law of $\mathcal{C}$ (or equivalently $\mathcal{C}^{\prime}$ ), but it does not decay with the distance between $\mathcal{C}$ and $\mathcal{C}^{\prime}$. In the phase $K \gg J$, the expectation value of $\tilde{O}_{\mathcal{C}, \mathcal{C}^{\prime}}^{(1)}$ would decay exponentially with the distance between $\mathcal{C}$ and $\mathcal{C}^{\prime}$, and also exponentially with the area of $\mathcal{C}\left(\right.$ or $\left.\mathcal{C}^{\prime}\right)$.

It is unknown whether model Eq. 32 has a second order transition between the two phases mentioned above or not. But again we can embed the models into a parent model with $U(1)^{(1)}$ and $\tilde{U}(1)^{(1)}$ symmetries. For instance, the $H_{d}$ in Eq. 32 can be embedded into

$$
H_{d}=\int d^{3} \tilde{x} \sum_{\mu} \frac{U}{2} \hat{e}_{\tilde{\mathbf{x}}, \hat{\mu}}^{2}-t \cos \left(\nabla_{z}\left(\nabla_{x} \hat{a}_{y}-\nabla_{y} \hat{a}_{x}\right)\right)+(\text { permute } x, y, z)-g \cos \left(2 \hat{a}_{\mu}\right) .
$$

$\hat{e}$ and $\hat{a}$ are defined on the dual lattice links $(\tilde{\mathbf{x}}, \hat{\mu})$, which are also the plaquettes of the original cubic lattice (Fig. 22). This model admits a gapless phase described by the following action:

$$
\mathcal{S}_{d}=\int d^{3} \tilde{x} d \tau \frac{1}{2 U}\left(\partial_{\tau} \vec{a}\right)^{2}+\frac{t}{2}\left(\nabla_{z}\left(\nabla_{x} a_{y}-\nabla_{y} a_{x}\right)\right)^{2}+(\text { permute } x, y, z) .
$$

In this gapless phase, the ODO Eq. 34 becomes

$$
\tilde{O}_{\mathcal{C}, \mathcal{C}^{\prime}}^{(1)}=\prod_{\tilde{l} \in \mathcal{C}} \tau_{\tilde{l}}^{3} \prod_{\tilde{l} \in \mathcal{C}^{\prime}} \tau_{\tilde{l}}^{3} \sim \exp \left(\mathrm{i} \oint_{\mathcal{C}} \hat{a}_{\mu} d x^{\mu}\right) \exp \left(-\mathrm{i} \oint_{\mathcal{C}^{\prime}} \hat{a}_{\nu} d x^{\nu}\right) .
$$

The expectation value of $\tilde{O}_{\mathcal{C}, \mathcal{C}^{\prime}}^{(1)}$ can be evaluated with the continuum limit action Eq. 37 .

Our goal is to show that, the behavior of $\tilde{O}_{\mathcal{C}, \mathcal{C}^{\prime}}^{(1)}$ is different from the gapped phases. This effect can be readily shown by considering the simple case when both $\mathcal{C}$ and $\mathcal{C}^{\prime}$ are unit plaquettes in the XY planes, separated in the $z$ direction by distance $Z$. Then

$$
\begin{aligned}
\tilde{O}_{\mathcal{C}, \mathcal{C}^{\prime}}^{(1)}(Z) & \sim \exp \left(\left\langle\left(\nabla_{x} \hat{a}_{y}-\nabla_{y} \hat{a}_{x}\right)_{z=0}\left(\nabla_{x} \hat{a}_{y}-\nabla_{y} \hat{a}_{x}\right)_{z=Z}\right\rangle\right) \\
& \sim \exp \left(-c_{1} \sqrt{\frac{U}{t}} \log Z\right) \sim \frac{1}{|Z|^{2 \Delta_{\mathcal{C}, \mathcal{C}^{\prime}}}}, \quad \Delta_{\mathcal{C}, \mathcal{C}^{\prime}} \sim \sqrt{\frac{U}{t}}
\end{aligned}
$$


This power-law decay along the $z$ direction originates from the fact that the correlation function $\left\langle\left(\nabla_{x} \hat{a}_{y}-\right.\right.$ $\left.\left.\nabla_{y} \hat{a}_{x}\right)_{z=0}\left(\nabla_{x} \hat{a}_{y}-\nabla_{y} \hat{a}_{x}\right)_{z=Z}\right\rangle$ has a singularity $1 / k_{z}$ in the momentum space near $k_{z}=0$. This power-law scaling along $z$ is already very different from the expectation value of $\tilde{O}_{\mathcal{C}, \mathcal{C}^{\prime}}^{(1)}(Z)$ in the gapped phases of the models in Eq. 32. We also made efforts to compute $\tilde{O}_{\mathcal{C}, \mathcal{C}^{\prime}}^{(1)}$ for $\mathcal{C}, \mathcal{C}^{\prime}$ with more general shapes, this calculation is presented in the appendix.

To evaluate $O_{\mathcal{C}}^{(1)}$, again it is more convenient to use a third dual description of the theory:

$$
H_{d 2}=\int d^{3} x \frac{U}{2}\left(\nabla_{x} \nabla_{y}\left(\hat{\phi}_{x}(\mathbf{x})-\hat{\phi}_{y}(\mathbf{x})\right)\right)^{2}-t \cos \left(\hat{N}_{z}(\mathbf{x})\right)+(\text { permute } x, y, z)
$$

The operators in Eq. 40 are related to the operators in Eq. 36 through the mapping (the duality between $H_{d}$ and $H_{d 2}$ was first discussed in Ref. 21)

$$
\begin{aligned}
& \hat{e}_{\tilde{\mathbf{x}}, \hat{z}}=\nabla_{x} \nabla_{y}\left(\hat{\phi}_{x}(\mathbf{x})-\hat{\phi}_{y}(\mathbf{x})\right), \text { and permutation of } x, y, z . \\
& \nabla_{z}\left(\nabla_{x} \hat{a}_{\tilde{\mathbf{x}}, \hat{y}}-\nabla_{y} \hat{a}_{\tilde{\mathbf{x}}, \hat{x}}\right)=-\hat{N}_{z}(\mathbf{x}), \text { and permutation of } x, y, z .
\end{aligned}
$$

The gapless phase is described by the following action:

$$
\mathcal{S}_{d 2}=\int d^{3} x d \tau \frac{U}{2}\left(\nabla_{x} \nabla_{y}\left(\phi_{x}-\phi_{y}\right)\right)^{2}+\frac{1}{2 t}\left(\partial_{\tau} \phi_{z}\right)^{2}+(\text { permute } x, y, z)
$$

$\hat{\phi}_{i}(\mathbf{x})$ and $\hat{N}_{i}(\mathbf{x})$ are three pairs of conjugate variables defined on the sites of the original cubic lattice $\mathbf{x}$. Let us assume that the loop $\mathcal{C}$ in $O_{\mathcal{C}}^{(1)}$ is a rectangle in the XY plane, then

$$
\begin{aligned}
O_{\mathcal{C}}^{(1)} & =\prod_{l \in \mathcal{C}} \sigma_{l}^{3}=\prod_{(\tilde{\mathbf{x}}, \hat{z}) \in \mathcal{A}} \tau_{\tilde{\mathbf{x}}, \hat{z}}^{1}=\prod_{(\tilde{\mathbf{x}}, \hat{z}) \in \mathcal{A}} \exp \left(\mathrm{i} \pi \hat{e}_{\tilde{\mathbf{x}}, \hat{z}}\right) \\
& =\prod_{\mathbf{x} \in \mathcal{A}} \exp \left(\mathrm{i} \pi \nabla_{x} \nabla_{y}\left(\hat{\phi}_{x}(\mathbf{x})-\hat{\phi}_{y}(\mathbf{x})\right)\right)=\exp \left(\mathrm{i} \pi \sum_{\mathbf{x} \in \mathcal{A}} \nabla_{x} \nabla_{y}\left(\hat{\phi}_{x}(\mathbf{x})-\hat{\phi}_{y}(\mathbf{x})\right)\right) \\
& =\exp \left(\mathrm{i} \pi\left(\hat{\phi}_{x}(0,0)-\hat{\phi}_{x}(x, 0)-\hat{\phi}_{x}(0, y)+\hat{\phi}_{x}(x, y)\right)-\mathrm{i} \pi\left(\hat{\phi}_{y}(0,0)-\hat{\phi}_{y}(x, 0)-\hat{\phi}_{y}(0, y)+\hat{\phi}_{y}(x, y)\right)\right) .
\end{aligned}
$$

Again since our goal is to show that $O_{\mathcal{C}}^{(1)}$ has different behavior from the two gapped phases $K \gg J$ and $K \ll J$, it is sufficient to consider a special "narrow rectangular" shape of $\mathcal{C}$, i.e. $y=1$, but $x \gg 1$. $\left\langle O_{\mathcal{C}}^{(1)}\right\rangle$ in this case is evaluated as $\exp \left(\pi^{2}\left\langle\nabla_{y}\left(\phi_{x}-\phi_{y}\right)_{0,0} \nabla_{y}\left(\phi_{x}-\phi_{y}\right)_{x, 0}\right\rangle\right)$. The key correlation function $\left\langle\nabla_{y}\left(\phi_{x}-\phi_{y}\right)_{0,0} \nabla_{y}\left(\phi_{x}-\phi_{y}\right)_{x, 0}\right\rangle$ has an infrared singularity as $1 /\left|k_{x}\right|$ near $k_{x}=0 . O_{\mathcal{C}}^{(1)}$ with a narrow rectangular $\mathcal{C}$ scales as

$$
\left\langle O_{\mathcal{C}}^{(1)}\right\rangle \sim \frac{1}{|x|^{\Delta_{\mathcal{C}}}}, \quad \Delta_{\mathcal{C}} \sim \sqrt{\frac{t}{U}}
$$

The power law decay of $O_{\mathcal{C}}^{(1)}$ is very different from the two gapped phases of Eq. 32. The results of this subsection are summarized in the table below.

\begin{tabular}{|c|c|c|c|}
\hline Special $3 d Z_{2}$ Gauge theory Eq. 32 & $K \gg J$ & $K \ll J$ & Gapless Phase \\
\hline$O_{\mathcal{C}}^{(1)}$ with rect. $\mathcal{C}$ in XY & Corner law & Area law & $\frac{1}{|x|^{\Delta_{\mathcal{C}}}}$, with $y=1$ and $x \gg 1$. \\
\hline$\tilde{O}_{\mathcal{C}, \mathcal{C}^{\prime}}^{(1)}$ parallel $\mathcal{C}, \mathcal{C}^{\prime}$ in XY; & Area law of $\mathcal{C}, \mathcal{C}^{\prime} ;$ & Perimeter law of $\mathcal{C} ;$ & $\frac{1}{|Z|_{\mathcal{C}, \mathcal{C}^{\prime}}}$, for unit square \\
separated along $\hat{z}$ & exponential decay with $Z$ & long range with $Z$ & $\mathcal{C}, \mathcal{C}^{\prime}$ separated along $z$ \\
\hline
\end{tabular}

\section{SUMMARY}

In this manuscript we analyzed the behavior of order diagnosis operators (ODO), at fine-tuned critical points or gapless phases of lattice systems with microscopic discrete categorical symmetries. The symmetries on both sides of the duality of the lattice models are constituents of the entire categorical symmetry of the system. We demonstrate that at these selected criticalities, the behavior of ODOs of categorical symmetries can be evaluated analytically, and 
they could have rather different scalings from the gapped phases of these models, where the ODO can be computed using the perturbation theory. The existence of subsystem symmetries of some of the systems intrinsically modify the behavior of ODOs at both the gapped phases, and the criticalities. And in examples with subsystem symmetries, we found that at these criticalities the scaling of ODOs defined on both sides of the duality of the lattice models is substantially different from the gapped phases.

While preparing for our manuscript, we became aware of a work that numerically computed the behavior of ODO of $\tilde{Z}_{2}^{(1)}$ at the $3 D$ Ising critical point, combined with theoretical comparison with free field theories 2 . The conclusion in this work is that, the $\tilde{Z}_{2}^{(1)}$ symmetry is still spontaneously broken at the $3 D$ Ising critical point. The conclusion is similar to ours at the fine-tuned Lifshitz criticality of $2 d$ lattice quantum Ising systems.

This work is supported by NSF Grant No. DMR-1920434, the David and Lucile Packard Foundation, and the Simons Foundation. The authors thank Chao-Ming Jian for helpful discussions.

\section{Appendix A: More calculations for $\tilde{O}_{\mathcal{C}, \mathcal{C}^{\prime}}^{(1)}$}

Let's first analyze the expectation value of $\tilde{O}_{\mathcal{C}, \mathcal{C}^{\prime}}^{(1)}$ defined in Eq. 34 which can be calculated using the continuous gauge theory Eq. 37 via

$$
\left\langle\widetilde{O}_{\mathcal{C}, \mathcal{C}^{\prime}}^{(1)}\right\rangle \sim \exp \left[\left(\oint_{\mathcal{C}} \oint_{\mathcal{C}^{\prime}}-\frac{1}{2} \oint_{\mathcal{C}} \oint_{\mathcal{C}}-\frac{1}{2} \oint_{\mathcal{C}^{\prime}} \oint_{\mathcal{C}^{\prime}}\right)\left\langle a_{\mu}(\mathbf{x}) a_{\nu}\left(\mathbf{x}^{\prime}\right)\right\rangle d x^{\mu} d x^{\prime \nu}\right] .
$$

With a Faddeev-Popov type gauge fixing $\zeta$ term, the gauge field propagator $D_{\mu \nu}(\omega, \mathbf{k})$ is given by

$$
D_{\mu \nu}^{-1}(\omega, \mathbf{k})=\left(\begin{array}{ccc}
\omega^{2} / U+2 t k_{y}^{2} k_{z}^{2} & -t k_{x} k_{y} k_{z}^{2} & -t k_{x} k_{z} k_{y}^{2} \\
-t k_{x} k_{y} k_{z}^{2} & \omega^{2} / U+2 t k_{z}^{2} k_{x}^{2} & -t k_{y} k_{z} k_{x}^{2} \\
-t k_{x} k_{z} k_{y}^{2} & -t k_{y} k_{z} k_{x}^{2} & \omega^{2} / U+2 t k_{x}^{2} k_{y}^{2}
\end{array}\right)-\zeta^{-1} k_{\mu} k_{\nu} .
$$

Our gauge choice is $\zeta \rightarrow 0$. Since we are interested in the expectation value of ODO of a static state, we will use the equal time Green's function. Directly using the full form of $D_{\mu \nu}$ would be tedious, but we observe that $D_{x x}$ has linear singularity at $k_{y} \rightarrow 0$, and $k_{z} \rightarrow 0$, which will dominate IR behavior of the Green's function. We can extract the most singular part of the Green's function, then $D_{x x}$ at $\tau=0$ reads

$$
D_{x x}(\tau=0, \mathbf{k})=\int \frac{d \omega}{2 \pi} D_{x x}(\omega, \mathbf{k})=\sqrt{\frac{U}{t}} \frac{1}{\sqrt{6}}\left(\frac{k_{y}^{2}}{\left(k_{x}^{2}+k_{y}^{2}\right)^{3 / 2}} \frac{1}{\left|k_{z}\right|}+\frac{k_{z}^{2}}{\left(k_{x}^{2}+k_{z}^{2}\right)^{3 / 2}} \frac{1}{\left|k_{y}\right|}\right)+\cdots
$$

This approximate form of Green's function captures the singularity at $k_{y} \rightarrow 0$ and $k_{z} \rightarrow 0$ separately. There is an extra singularity when multiple momenta approach zero simultaneously. But since this extra singularity occurs at a much smaller measure of the momentum space compared with the singularities captured by Eq. A3, we take the approximate form of Green's function Eq. A3. Further analysis may be demanded to address all singularities in the Green's function.

Similarly, we approximate the off-diagonal term $D_{x y}$ around its singularity $k_{z}=0$

$$
D_{x y}(\tau=0, \mathbf{k})=\sqrt{\frac{U}{t}} \frac{-k_{x} k_{y}}{\sqrt{6}\left(k_{x}^{2}+k_{y}^{2}\right)^{3 / 2}} \frac{1}{\left|k_{z}\right|}+\cdots
$$

Other components of $D_{\mu \nu}$ can be obtained by the permutations of $k_{x}, k_{y}, k_{z}$. The real-space expression of the Green's function is then obtained through Fourier transformation:

$$
D_{\mu \nu}(\tau=0, \mathbf{x})=\sqrt{\frac{U}{t}} \frac{-1}{2 \sqrt{6} \pi^{2}}\left(\begin{array}{ccc}
\frac{x^{2} \log |z \delta|}{\left(x^{2}+y^{2}\right)^{3 / 2}}+\frac{x^{2} \log |y \delta|}{\left(x^{2}+z^{2}\right)^{3 / 2}} & \frac{x y \log |z \delta|}{\left(x^{2}+y^{2}\right)^{3 / 2}} & \frac{x z \log |y \delta|}{\left(x^{2}+z^{2}\right)^{3 / 2}} \\
\frac{x y \log \mid z \delta \delta}{\left(x^{2}+y^{2}\right)^{3 / 2}} & \frac{y^{2} \log |x \delta|}{\left(y^{2}+z^{2}\right)^{3 / 2}}+\frac{y^{2} \log |z \delta|}{\left(y^{2}+x^{2}\right)^{3 / 2}} & \frac{y z \log |x \delta|}{\left(y^{2}+z^{2}\right)^{3 / 2}} \\
\frac{x z \log |y \delta|}{\left(x^{2}+z^{2}\right)^{3 / 2}} & \frac{y z \log |x \delta|}{\left(y^{2}+z^{2}\right)^{3 / 2}} & \frac{z^{2} \log |y \delta|}{\left(z^{2}+x^{2}\right)^{3 / 2}}+\frac{z^{2} \log |x \delta|}{\left(z^{2}+y^{2}\right)^{3 / 2}}
\end{array}\right),
$$

where $\delta>0$ is a small IR cut-off, which is needed in the Fourier transformation of $1 /|k|$. This is the effective real-space Green function that can be used to calculate the scaling behaviors of $\left\langle\tilde{O}_{\mathcal{C}, \mathcal{C}^{\prime}}^{(1)}\right\rangle$. 
Let's consider two identical squares $\mathcal{C}, \mathcal{C}^{\prime}$ that are completely parallel to each other. We assume $\mathcal{C}$ has four corners $(0,0,0),(L, 0,0),(L, L, 0),(0, L, 0)$, and $\mathcal{C}^{\prime}$ has four corners $(0,0, Z),(L, 0, Z),(L, L, Z),(0, L, Z)$. Based on the realspace Green function Eq. A5, an integral over $\mathcal{C}, \mathcal{C}^{\prime}$ leads to

$$
-\log \left\langle\tilde{O}_{\mathcal{C}, \mathcal{C}^{\prime}}^{(1)}\right\rangle=\sqrt{\frac{U}{t}} \frac{4 L}{\sqrt{6} \pi^{2}}\left(\begin{array}{c}
\left(2\left(\sqrt{L^{2}+Z^{2}}-Z\right) / L+\log \left(\sqrt{L^{2}+Z^{2}}-L\right)\right) \log (L / \epsilon)+\log L(\log L-3 \log \epsilon) \\
-\log (L Z)+\left(\sqrt{2}-\sinh ^{-1}(1)\right) \log (Z / \epsilon)+2 \log \epsilon(\log \epsilon+1)
\end{array}\right) .
$$

where $\epsilon>0$ is a small UV cut-off. It is important to notice that, although the real space Green's function has a dependence on the IR cut-off $\delta$, the final result of $\tilde{O}_{\mathcal{C}, \mathcal{C}^{\prime}}^{(1)}$ is free from any IR-divergence. We are most interested in the behaviors of $\left\langle\tilde{O}_{\mathcal{C}, \mathcal{C}^{\prime}}^{(1)}\right\rangle$ under the large- $L$ and large- $Z$ limits:

$$
\left\langle\tilde{O}_{\mathcal{C}, \mathcal{C}^{\prime}}^{(1)}\right\rangle \sim\left\{\begin{array}{ll}
e^{-\sqrt{\frac{U}{t}} \frac{4}{\sqrt{6} \pi^{2}} L\left(\log (L / \epsilon)+\sqrt{2}-1-\sinh ^{-1}(1)\right) \log Z}=e^{-c_{1} \sqrt{\frac{U}{t}} \log Z} & L<+\infty, Z \rightarrow+\infty \\
e^{-\sqrt{\frac{U}{t}} \frac{4}{\sqrt{6} \pi^{2}}(2 \log (Z / \epsilon)+1-\log 2) L \log L}=e^{-c_{2} \sqrt{\frac{U}{t}} L \log L} & Z<+\infty, L \rightarrow+\infty
\end{array},\right.
$$

where $c_{1}$ and $c_{2}$ are two numerical coefficients which depend on the UV cut-off $\epsilon$.

${ }^{1}$ W. Ji and X.-G. Wen, Phys. Rev. Research 2, 033417 (2020), URL https://link.aps.org/doi/10.1103/PhysRevResearch. 2.033417 .

2 O. Aharony, N. Seiberg, and Y. Tachikawa, Journal of High Energy Physics 2013 (2013), ISSN 1029-8479, URL http: //dx.doi.org/10.1007/JHEP08(2013)115

3 S. Gukov and A. Kapustin, Topological quantum field theory, nonlocal operators, and gapped phases of gauge theories (2013), 1307.4793.

4 A. Kapustin and R. Thorngren, Topological field theory on a lattice, discrete theta-angles and confinement (2013), 1308.2926.

5 A. Kapustin and R. Thorngren, Higher symmetry and gapped phases of gauge theories (2013), 1309.4721.

6 A. Kapustin and N. Seiberg, Journal of High Energy Physics 2014 (2014), ISSN 1029-8479, URL http://dx.doi.org/10. 1007/JHEP04 (2014)001

7 D. Gaiotto, A. Kapustin, N. Seiberg, and B. Willett, Journal of High Energy Physics 2015 (2015), ISSN 1029-8479, URL http://dx.doi.org/10.1007/JHEP02(2015) 172

8 P.-S. Hsin, H. T. Lam, and N. Seiberg, SciPost Physics 6 (2019), ISSN 2542-4653, URL http://dx.doi.org/10.21468/ SciPostPhys.6.3.039

9 N. Seiberg, SciPost Physics 8 (2020), ISSN 2542-4653, URL http://dx.doi.org/10.21468/SciPostPhys.8.4.050

10 C. Córdova, T. T. Dumitrescu, and K. Intriligator, Journal of High Energy Physics 2019, 184 (2019), 1802.04790.

11 E. Fradkin and L. Susskind, Phys. Rev. D 17, 2637 (1978), URL https://link.aps.org/doi/10.1103/PhysRevD.17.2637

12 C. L. Henley, Journal of Physics: Condensed Matter 16, S891CS898 (2004), ISSN 1361-648X, URL http://dx.doi.org/ 10.1088/0953-8984/16/11/045

13 E. Fradkin, D. A. Huse, R. Moessner, V. Oganesyan, and S. L. Sondhi, Physical Review B 69 (2004), ISSN 1550-235X, URL http://dx.doi.org/10.1103/PhysRevB.69.224415

14 D. S. Rokhsar and S. A. Kivelson, Phys. Rev. Lett. 61, 2376 (1988), URL https://link.aps.org/doi/10.1103/ PhysRevLett.61.2376.

15 E. Fradkin and S. Kivelson, Modern Physics Letters B 04, 225 (1990).

16 J. B. Kogut, Rev. Mod. Phys. 51, 659 (1979), URL https://link.aps.org/doi/10.1103/RevModPhys.51.659

17 R. Savit, Rev. Mod. Phys. 52, 453 (1980), URL https://link.aps.org/doi/10.1103/RevModPhys.52.453

18 C. Xu and L. Fu, Phys. Rev. B 81, 134435 (2010), URL https://link.aps.org/doi/10.1103/PhysRevB.81.134435

19 C. Xu and J. E. Moore, Physical Review Letters 93 (2004), ISSN 1079-7114, URL http://dx.doi.org/10.1103/ PhysRevLett.93.047003.

20 A. Paramekanti, L. Balents, and M. P. A. Fisher, Physical Review B 66 (2002), ISSN 1095-3795, URL http://dx.doi.org/ 10.1103/PhysRevB.66.054526

21 C. Xu and M. P. A. Fisher, Physical Review B 75 (2007), ISSN 1550-235X, URL http://dx.doi.org/10.1103/PhysRevB. 75.104428

22 J. Zhao, Z. Yan, M. Cheng, and Z. Y. Meng, Higher-form symmetry breaking at ising transitions (2020), 2011.12543.

23 The expectation value of ODOs should not be viewed as an analogue of order parameter, they should be viewed as analogue of correlation of order parameters. The ODOs were studied as the "patch symmetry operators" of the categorical symmetry in Ref. 1 .

24 Spontaneous breaking of emergent higher form symmetries in the infrared would still lead to gapless Goldstone modes, this is very different from the scenario of ordinary 0 -form symmetries. 